\title{
Stay safe stay connected: surgical mobile app at the time of Covid-19 outbreak
}

\author{
Giuliano Barugola $^{1} \cdot$ Elisa Bertocchi $^{1}$ (D) - Giacomo Ruffo ${ }^{1}$
}

Accepted: 14 May 2020 / Published online: 25 May 2020

(C) Springer-Verlag GmbH Germany, part of Springer Nature 2020

\section{Dear Editor:}

The coronavirus has placed the healthcare worldwide system under tremendous strain. As part of the global approach to manage COVID-19, a number of social distancing measures were implemented. In the health context, the good practices should consider delaying or re-scheduling patients coming in both for not urgent situations and periodic exams, in particular for the elderly or those with chronic health issues [1]. Not only for patients' safety but also to ensure resources, the Italian National Institute of Health has recommended that nonemergency surgery be delayed. Moreover, it must be considered that COVID-19 can complicate the perioperative course with diagnostic challenge and a high potential fatality rate. In locations with widespread infections and limited resources, the risk of elective surgical procedures may outweigh the benefit [2]. The authors have expertise in colorectal surgery, and they have changed the day-life practice concerning not only the surgery itself but also the extensive management of patients with colorectal conditions. From March 1 to April 25, 2020 , we have rescheduled $82 \%$ of colorectal resections for benign conditions. On the contrary only $12 \%$ for malignancies has been postponed because the delay could have had an impact on the quality of care and condition a worsening of results even in the long term of cancer treatment [3]. During the same time period, we have also reduced about $60 \%$ of outpatient clinic for patients with stoma and colorectal diseases but there are some conditions that even if not cancer related, cannot be deferred (e.g. the stoma education and management). Recently, some authors have underlined pragmatical and technical suggestions in the work-up for colorectal surgical patients during Covid-19 outbreak but remains poorly

Elisa Bertocchi

elisa.bertocchi@sacrocuore.it

1 General Surgery Unit, IRCCS Ospedale Sacro Cuore Don Calabria, Via Don A. Sempreboni 5, 37024, Negrar di Valpolicella, Verona, Italy investigated the way to follow step by step the patient during the perioperative period [4].

For these reasons, we have searched for a smart tool to start many remote consultations as soon as possible, replacing patient visits.

During these hours, the authorities began to look ahead to a second phase in which a sudden resumption of surgical activity will be inevitable but will clash with the persistent respect for measures of social distance.

The Operative Unit of General Surgery of IRCCS Sacro Cuore Don Calabria Hospital of Negrar di Valpolicella (VR) is speeding up the launch of a new mobile app, named iColon, that enables people to easily and safely evaluate their conditions. iColon is available on smartphones and tablets operating iOS 11.1 and android 5.0 and up. The platform will help patients in the route of surgery based on ERAS guidelines ensuring customized and real-optimized care [5]. Moreover, patients will be able to receive some of the customized postsurgical care they need without having to go to a medical centre. iColon sends push notifications twice daily to remember to fill out the adhesion of the items. At the end of each daily questionnaire, the app provides a feedback to clinicians on the patient's status and adherence to daily optimization and recovery after discharge. The surgeon follows patients' route on a dedicated web-app platform. In case of critical issues arbitrarily chosen for each phase such as fever, increased stoma output and difficult pain control, a specific alert is sent to the surgeons. For the abovementioned reasons, the IRCCS Sacro Cuore Don Calabria Hospital has designed the iColon app, customized during the outbreak to support government efforts to reduce the spread of the disease. In the COVID-19 era, iColon will connect patients with their care team and will facilitate the take-over of needful information about patient's health allowing a safe hospitalization.

According to the authorities, the surgical team, thanks to iColon, will verify the patient's COVID-19 checklist before hospital access. In addition, after discharge, the app could aid the process of identifying people who have been developed 
suspicious symptoms and their potential "contacts". While many patients will understand the circumstances and the need for this evolution, there will be challenges associated with a rapid transition to an outpatient telehealth. At first, the sacred patient-physician relationship has traditionally been developed during face-to-face interactions which will represent a shift from our current culture. Secondly, some patients may not have access to required technology for telehealth services, which may exacerbate disparities in obtaining care during the current pandemic.

To maintain complete and effective healthcare, the connection between patient and surgeon is critical to ensuring treatment. These problems have become a topic of debate as much of the world faces a blockage during the outbreak of COVID19. Mobile technologies could provide a valuable tool to help patients stay in touch with doctors during the preoperative period and after discharge.

\section{Compliance with ethical standards}

Conflict of interest The authors declare that they have no conflict of interest.

\section{References}

1. Wee LE, Conceicao EP, Sim XYJ, Aung MK, Tan KY, Wong HM, Wijaya L, Tan BH, Ling ML, Venkatachalam I (2020) Minimising intra-hospital transmission of COVID-19: the role of social distancing. J Hosp Infect 2 pii S0195-6701(20):30191-30192. https://doi. org/10.1016/j.jhin.2020.04.016

2. COVIDSurg Collaborative (2020) Global guidance for surgical care during the COVID-19 pandemic. Br J Surg doi. https://doi.org/10. 1002/bjs. 11646

3. Pellino G, Spinelli A (2020) How COVID-19 outbreak is impacting colorectal cancer patients in Italy: a long shadow beyond infection. Dis Colon Rectum doi 63:720-722. https://doi.org/10.1097/DCR. 0000000000001685

4. Lisi G, Campanelli M, Spoletini D, Carlini M (2020) The possible impact of COVID-19 on colorectal surgery in Italy. Colorectal Dis. https://doi.org/10.1111/codi.15054

5. Pecorelli N, Fiore JF Jr, Kaneva P, Somasundram A, Charlebois P, Liberman AS, Stein BL, Carli F, Feldman LS (2018) An app for patient education and self-audit within an enhanced recovery program for bowel surgery: a pilot study assessing validity and usability. Surg Endosc 32:2263-2273. https://doi.org/10.1007/s00464-017$5920-3$

Publisher's note Springer Nature remains neutral with regard to jurisdictional claims in published maps and institutional affiliations. 